\title{
H9N2 influenza virus acquires intravenous pathogenicity on the introduction of a pair of di-basic amino acid residues at the cleavage site of the hemagglutinin and consecutive passages in chickens
}

\author{
Kosuke Soda', Shingo Asakura', Masatoshi Okamatsu', Yoshihiro Sakoda', Hiroshi kida 1,2,3*
}

\begin{abstract}
Background: Outbreaks of avian influenza (Al) caused by infection with low pathogenic H9N2 viruses have occurred in poultry, resulting in serious economic losses in Asia and the Middle East. It has been difficult to eradicate the H9N2 virus because of its low pathogenicity, frequently causing in apparent infection. It is important for the control of Al to assess whether the H9N2 virus acquires pathogenicity as $\mathrm{H} 5$ and $\mathrm{H} 7$ viruses. In the present study, we investigated whether a non-pathogenic H9N2 virus, A/chicken/Yokohama/aq-55/2001 (Y55) (H9N2), acquires pathogenicity in chickens when a pair of di-basic amino acid residues is introduced at the cleavage site of its HA molecule.
\end{abstract}

Results: rgY55sub (H9N2), which had four basic amino acid residues at the HA cleavage site, replicated in MDCK cells in the absence of trypsin after six consecutive passages in the air sacs of chicks, and acquired intravenous pathogenicity to chicken after four additional passages. More than $75 \%$ of chickens inoculated intravenously with the passaged virus, rgY55sub-P10 (H9N2), died, indicating that it is pathogenic comparable to that of highly pathogenic avian influenza viruses (HPAIVs) defined by World Organization for Animal Health (OIE). The chickens inoculated with the virus via the intranasal route, however, survived without showing any clinical signs. On the other hand, an avirulent H5N1 strain, A/duck/Hokkaido/Nac-1/2004 (Vac1) (H5N1), acquired intranasal pathogenicity after a pair of di-basic amino acid residues was introduced into the cleavage site of the HA, followed by two passages by air sac inoculation in chicks.

Conclusion: The present results demonstrate that an H9N2 virus has the potential to acquire intravenous pathogenicity in chickens although the morbidity via the nasal route of infection is lower than that of H5N1 HPAIV.

\section{Background}

Each of the known subtypes of the influenza A virus (H1 to H16 and N1 to N9) is circulating in water birds, especially in migratory ducks [1]. A highly pathogenic avian influenza virus (HPAIV) is generated when a nonpathogenic virus brought in by migratory birds from nesting lakes in the north is transmitted to chickens via

\footnotetext{
* Correspondence: kida@vetmed.hokudai.ac.jp

'Laboratory of Microbiology, Department of Disease Control, Graduate School of Veterinary Medicine, Hokkaido University, Sapporo, Hokkaido 0600818, Japan

Full list of author information is available at the end of the article
}

domestic ducks, geese, quails, turkeys, etc. and acquires pathogenicity for chickens with repeated multiple infections in the chicken population [2-6]. The hemagglutinins (HAs) of HPAIVs differ from those of low pathogenic avian influenza viruses (LPAIVs) with a pair of di-basic amino acid residues at their cleavage site [7]. This structure permits ubiquitous proteases such as furin and PC6, which recognize multiple basic amino acids, to cleave the HA, leading to systemic infection in chickens. By contrast, HAs of LPAIVs are cleaved only by trypsin-like proteases which are expressed in the cells lining the respiratory or intestinal tracts, so that the 
viruses cause only localized infections, resulting in mild or asymptomatic diseases. It is presently believed that the strains only with $\mathrm{H} 5$ or H7 HAs become HPAIVs during extensive infections in chicken populations [8]. The reason why the subtypes of HPAIVs are restricted to $\mathrm{H} 5$ and $\mathrm{H} 7$ is not known although a model demonstrating that $\mathrm{H} 5 \mathrm{HA}$ is cleaved by furin through molecular docking analyses have been proposed $[9,10]$.

H9N2 avian influenza virus strains have caused outbreaks in poultry, resulting in serious economic losses in Asia and the Middle East [11-19]. The causal strains, however, are avirulent and none of them have multiple basic amino acid residues at the cleavage site of the HA $[12,15]$. No specific-pathogen-free chickens experimentally infected with H9N2 isolates from diseased chickens showed any clinical symptoms [20]. Co-infection of H9N2 viruses with bacteria such as Staphylococcus aureus and Haemophilus paragallinarum or with attenuated coronavirus vaccine exacerbated the disease [19,21-23].

Since H9N2 viruses have been isolated not only from domestic birds but also from pigs and humans, the H9 virus has the potential to cause a next pandemic in humans [17,24-27]. It is important for controlling avian influenza and for preparing for pandemic influenza to assess whether the H9N2 virus aquires pathogenicity as $\mathrm{H} 5$ and $\mathrm{H} 7$ viruses. In the present study, we introduced a pair of di-basic amino acid residues into the cleavage site of the H9 and H5 HAs of non-pathogenic strains. These mutant $\mathrm{H} 9$ and $\mathrm{H} 5$ viruses were then serially passaged in the air sacs of chicks and their pathogenicity was assessed by inoculation to four-week-old chickens via intravenous and intranasal routes.

\section{Results}

\section{Generation and characterization of mutant viruses}

To investigate whether a non-pathogenic H9 influenza virus, A/chicken/Yokohama/aq-55/2001 (Y55) (H9N2) acquires pathogenicity on the introduction of a pair of di-basic amino acid residues at their HA cleavage site, rgY55sub (H9N2) was generated by site-directedmutagenesis and reverse genetics. Amino acid sequences at the HA cleavage site of the mutant strain are shown in Figure 1A. The RKKR motif was introduced into the H9 HA cleavage site to give a pair of di-basic amino acid residues that is known to be a sine qua non for $\mathrm{H} 5$ and $\mathrm{H} 7$ viruses to become highly pathogenic to chickens. The virus with the insertion of basic amino acid residues at the H9 HA cleavage site was not rescued from plasmidtransfected cells (data not shown). As a positive control, rgVac1ins (H5N1) was generated by inserting the RRKKR motif, rather than RKKR, into the HA of the non-pathogenic virus A/duck/Hokkaido/Vac-1/2004 (Vac1) (H5N1) since recent H5 HPAIV isolates have the motif as insertion mutation. rgVac1sub (H5N1) was also generated to

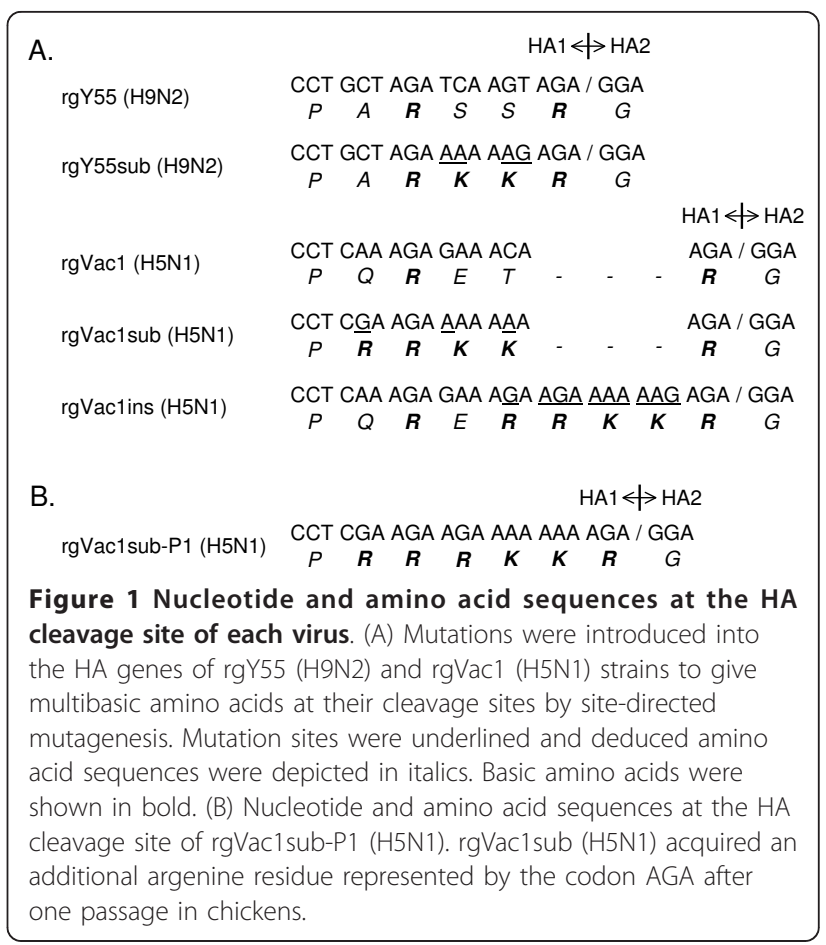

examine whether subtitution mutation with basic amino acid residues at the HA cleavage site contributed to acquisition of pathogenicity for chickens.

rgY55sub (H9N2) and rgVac1ins (H5N1) required trypsin to replicate in MDCK cells, and showed similar levels of growth to their parental viruses (Table 1). Chickens intravenously inoculated with rgY55sub (H9N2) or rgVac1ins (H5N1) did not show any signs of disease. rgVac1sub (H5N1) replicated in MDCK cells without exogenous trypsin, and one of the eight chickens inoculated with the virus showed slight depression at one day post-infection.

\section{Consecutive passages of the viruses in the air sacs of chicks}

The H9 mutant virus was serially passaged in the air sacs of chicks to assess whether it acquires pathogenicity as did $\mathrm{H} 5$ viruses. The passaged viruses were tested for their growth potential in MDCK cells and pathogenicity for chickens (Table 1). rgY55sub (H9N2) replicated in MDCK cells in the absence of trypsin and killed all of the chicks after six consecutive passages. Two of the eight four-week-old chickens inoculated intravenously with rgY55sub-P8 (H9N2) died within five days. Consequently, over $75 \%$ of the chickens intravenously infected with rgY55sub-P10 (H9N2) died by two days post inoculation, and its pathogenicity was comparable to that of the known HPAIVs [28].

H5N1 mutant viruses acquired intravenous pathogenicity by passaging twice in chicks; all of the chickens 
Table 1 Growth potential in MDCK cells and pathogenicity for chicken of each virus

\begin{tabular}{lcccc}
\hline Viruses & \multicolumn{2}{c}{ Plaque formation (log PFU/ml) } & \multicolumn{2}{c}{ Pathogenicity (number of dead/sick/total ) } \\
\cline { 2 - 5 } & With trypsin & Without trypsin & 3-day-old chicks (air sac inoculation) & 4-week-old chickens (intravenous inoculation) \\
\hline rgY55 (H9N2) & 8.1 & $-^{a}$ & $N^{b}$ & $0 / 0 / 8$ \\
rgY55sub (H9N2) & 8.0 & - & $0 / 0 / 3$ & $0 / 0 / 8$ \\
rgY55sub-P5 (H9N2) & 7.6 & - & $0 / 0 / 4$ & $0 / 0 / 8$ \\
rgY55sub-P6 (H9N2) & 7.2 & 6.7 & $3 / 3 / 3$ & $0 / 1 / 8$ \\
rgY55sub-P7 (H9N2) & 7.8 & 7.5 & $3 / 3 / 3$ & $0 / 5 / 8$ \\
rgY55sub-P8 (H9N2) & 7.6 & 7.6 & $3 / 3 / 3$ & $2 / 7 / 8(4.0)^{c}$ \\
rgY55sub-P9 (H9N2) & 7.2 & 6.9 & $3 / 3 / 3$ & $1 / 8 / 8(10.0)$ \\
rgY55sub-P10 (H9N2) & 6.5 & 6.1 & $3 / 3 / 3$ & $6 / 8 / 8(1.8)$ \\
& & & & $0 / 0 / 8$ \\
rgVac1 (H5N1) & 7.6 & - & $N T$ & $0 / 1 / 8$ \\
rgVac1sub (H5N1) & 7.6 & 7.8 & $0 / 0 / 3$ & $3 / 5 / 8(5.3)$ \\
rgVac1sub-P1 (H5N1) & 6.8 & 6.8 & $3 / 3 / 3$ & $8 / 8 / 8(2.6)$ \\
rgVac1sub-P2 (H5N1) & 6.4 & 6.5 & $6 / 6 / 6$ & $0 / 0 / 8$ \\
rgVac1ins (H5N1) & 7.3 & - & $0 / 0 / 3$ & $6 / 7 / 8(6.8)$ \\
rgVac1ins-P1 (H5N1) & 7.8 & 7.1 & $3 / 3 / 3$ & $6 / 8 / 8(4.4)$ \\
rgVac1ins-P2 (H5N1) & 7.1 & 7.1 & $4 / 4 / 4$ &
\end{tabular}

${ }^{a}$ A plaque was not observed.

b Not tested.

c Mean death days are shown in parentheses.

died after intravenous inoculation with rgVac1sub-P2 (H5N1) or rgVac1ins-P2 (H5N1).

Amino acid changes of the viruses during consecutive passages in the air sacs of chicks

Nucleotide sequences of the eight segmented genomes of the viruses passaged in the air sacs of chicks were analyzed and compared with those of each parental virus. Leu234 (equivalent to position 226 of H3 HA) in the HA of rgY55sub (H9N2) was substituted with glutamine at the initial passage (Table 2). No other amino acid change was observed up to the fifth passage. Four amino acids in the HA, NA and M2 changed at the sixth passage. One of the asparagine-linked glycosylation sites on the HA was lost by Asn29His mutation. In

Table 2 Amino acid changes during consecutive passages of rgY55sub (H9N2)

\begin{tabular}{|c|c|c|c|c|c|c|c|c|}
\hline \multirow[t]{2}{*}{ Passage number } & \multicolumn{2}{|l|}{ PB2 } & \multicolumn{3}{|c|}{$\mathrm{HA}$} & \multirow{2}{*}{$\frac{N P}{54}$} & \multirow{2}{*}{$\begin{array}{c}\text { NA } \\
195\end{array}$} & \multirow{2}{*}{$\frac{M 2}{51}$} \\
\hline & $271^{a}$ & 29 & 234 & 357 & 391 & & & \\
\hline PO & $\mathrm{T}$ & $\mathrm{N}$ & $L$ & $A$ & $\mathrm{~N}$ & W & $\mathrm{T}$ & 1 \\
\hline P1-P5 & $b$ & . & Q & . & . & . & . & . \\
\hline P6 & . & $\mathrm{H}$ & Q & D & . & . & A & $\mathrm{T}$ \\
\hline P7 & . & $\mathrm{H}$ & Q & D & $N / D^{c}$ & . & A & $\mathrm{T}$ \\
\hline P8 & $\mathrm{T} / \mathrm{A}$ & $H$ & Q & D & N/D & . & A & $\mathrm{T}$ \\
\hline P9 & $\mathrm{T} / \mathrm{A}$ & $\mathrm{H}$ & Q & D & $\mathrm{N} / \mathrm{D}$ & G & A & $\mathrm{T}$ \\
\hline P10 & $A$ & $\mathrm{H}$ & $Q$ & $D$ & D & $\mathrm{G}$ & $A$ & $\mathrm{~T}$ \\
\hline
\end{tabular}

\footnotetext{
${ }^{a}$ Methionine encoded by the AUG start codon is defined as position 1

b Periods indicate same amino acids as the parental virus.

c Amino acid quasispecies are observed.
}

total, eight amino acid differences were found between rgY55sub (H9N2) and rgY55sub-P10 (H9N2). Five and one amino acid changes were found in the PA, HA, M1 and M2 of rgVac1sub-P2 (H5N1), and the HA of rgVac1ins-P2 (H5N1), respectively (Table 3). It is worth noting that one argenine was inserted at the HA cleavage site of rgVac1sub (H5N1) after one passage in chickens (Figure 1B).

\section{Pathogenicity of the viruses on intranasal infection in chickens}

To examine whether the pathogenicity of each virus via the intranasal route of infection correlates with that via intravenous route, three 4-week-old chickens were intranasally inoculated with the viruses of $10^{6.5} 50 \%$ egg infectious dose $\left(\mathrm{EID}_{50}\right)$ and observed for clinical signs until day 14 post-infection (Table 4). All chickens inoculated with rgY55sub-P10 (H9N2) or its parental rgY55sub (H9N2) survived without showing any clinical signs, and serum antibodies were detected (1:128-2,048 HI titers), indicating that virus replication occurred.

One of the three chickens inoculated with rgVac1sub (H5N1) or rgVac1ins (H5N1) showed seroconversion after 14 days while no chickens were susceptible to infection with rgVac1 (H5N1). Both of rgVac1sub-P2 (H5N1) and rgVac1ins-P2 (H5N1) were pathogenic, killing two of the three chickens by day 11 post-inoculation.

Additional passages of the Vac1-based viruses in chickens One of the three chickens intranasally inoculated with rgVac1sub-P2 (H5N1) or rgVac1ins-P2 (H5N1) did not 
Table 3 Amino acid changes during consecutive passages of rgVac1 mutants

\begin{tabular}{|c|c|c|c|c|c|c|c|c|c|c|c|c|c|c|}
\hline \multirow[t]{3}{*}{ Passage number } & \multicolumn{9}{|c|}{ rgVac1sub (H5N1) } & \multicolumn{5}{|c|}{ rgVac1ins (H5N1) } \\
\hline & \multicolumn{2}{|c|}{ PA } & \multicolumn{2}{|c|}{ HA } & \multicolumn{2}{|c|}{ NP } & \multicolumn{2}{|c|}{ M1 } & \multirow{2}{*}{$\frac{\text { M2 }}{45}$} & \multicolumn{3}{|c|}{$\mathrm{HA}$} & \multirow{2}{*}{$\begin{array}{l}\text { NA } \\
171\end{array}$} & \multirow{2}{*}{$\begin{array}{l}\text { M1 } \\
130\end{array}$} \\
\hline & $65^{a}$ & 672 & 308 & 338 & 213 & 374 & 89 & 101 & & 157 & 298 & 465 & & \\
\hline PO & $\mathrm{S}$ & $\mathrm{L}$ & $\mathrm{H}$ & - & $\mathrm{R}$ & $M$ & D & $\mathrm{R}$ & $R$ & S & M & D & $\mathrm{N}$ & $\mathrm{L}$ \\
\hline P1 & b & . & Q & $\mathrm{R}^{c}$ & . & . & . & . & $\mathrm{H}$ & $P$ & 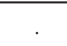 & $E$ & . & . \\
\hline P2 & $\mathrm{T}$ & . & Q & $\mathrm{R}$ & & & . & K & $\mathrm{H}$ & $P$ & . & & & . \\
\hline P3 & & $\mathrm{F}$ & Q & R & Q & V & N & K & $\mathrm{H}$ & $P$ & । & & $\mathrm{N} / \mathrm{H}^{\mathrm{d}}$ & । \\
\hline
\end{tabular}

${ }^{a}$ Methionine encoded by the AUG start codon is defined as position 1 .

${ }^{b}$ Periods indicate same amino acids as the parental virus.

c Arginine was inserted at the HA cleavage site.

d Amino acid quasispecies were observed.

show any clinical signs (Table 4), indicating that the viruses did not extensively replicate in chickens. rgVac1sub-P3 (H5N1) and rgVac1ins-P3 (H5N1) were prepared from the brain homogenates of the chickens that died on day 11 post-intranasal inoculation with the P2 viruses. Additional amino acid changes were found in P3 viruses (Table 3). To investigate whether the P3 viruses show higher pathogenicity in chicken, the viruses were inoculated via intranasal route. Mortality rate of chickens inoculated with the P3 viruses was equal to that with P2 viruses (Table 4).

\section{Growth potential of the H9N2 and H5N1 viruses in chickens}

To investigate whether tissue tropism of the viruses was involved in their pathogenicity, we determined viral

\begin{tabular}{|c|c|c|c|}
\hline $\begin{array}{l}\text { Inoculated } \\
\text { viruses }\end{array}$ & $\begin{array}{c}\text { Seroconversion at } \\
14 \text { d.p.i. }{ }^{a}\end{array}$ & $\begin{array}{l}\text { Clinical } \\
\text { signs }\end{array}$ & $\begin{array}{c}\text { Mortality (dead } \\
\text { days) }\end{array}$ \\
\hline rgY55 (H9N2) & $3 / 3^{b}$ & $0 / 3$ & $0 / 3$ \\
\hline $\begin{array}{l}\text { rgY55sub } \\
\text { (H9N2) }\end{array}$ & $3 / 3$ & $0 / 3$ & $0 / 3$ \\
\hline $\begin{array}{l}\text { rgY55sub-P10 } \\
\text { (H9N2) }\end{array}$ & $3 / 3$ & $0 / 3$ & $0 / 3$ \\
\hline rgVac1 (H5N1) & $0 / 3$ & $0 / 3$ & $0 / 3$ \\
\hline $\begin{array}{l}\text { rgVac1sub } \\
\text { (H5N1) }\end{array}$ & $1 / 3$ & $0 / 3$ & $0 / 3$ \\
\hline $\begin{array}{l}\text { rgVac1sub-P2 } \\
(\mathrm{H} 5 \mathrm{~N} 1)\end{array}$ & $0 / 1$ & $2 / 3$ & $2 / 3(4,11)$ \\
\hline $\begin{array}{l}\text { rgVac1sub-P3 } \\
\text { (H5N1) }\end{array}$ & $1 / 1$ & $3 / 3$ & $2 / 3(7,8)$ \\
\hline $\begin{array}{l}\text { rgVac1ins } \\
\text { (H5N1) }\end{array}$ & $1 / 3$ & $0 / 3$ & $0 / 3$ \\
\hline $\begin{array}{l}\text { rgVac1ins-P2 } \\
(\mathrm{H} 5 \mathrm{~N} 1)\end{array}$ & $1 / 1$ & $2 / 3$ & $2 / 3(8,11)$ \\
\hline $\begin{array}{l}\text { rgVac1ins-P3 } \\
\text { (H5N1) }\end{array}$ & $0 / 1$ & $2 / 3$ & $2 / 3(4,6)$ \\
\hline
\end{tabular}

${ }^{a}$ Examined for the survived chickens by $\mathrm{HI}$ test and ELISA.

${ }^{b}$ The number of positive animals/total. titers in the tissue and blood samples from four-weekold chickens intranasally inoculated with each virus on three days post infection (Table 5). rgY55 (H9N2) and rgVac1 (H5N1) were scarcely recovered from the samples, and the mutant strains before passage in chicks showed broader tissue tropism than the parental viruses. None of the chickens inoculated with rgY55sub-P10 (H9N2) showed any signs of disease, and viruses were recovered from each of the samples except the brain and the blood. One of the three chickens inoculated with rgVac1sub-P2 (H5N1) showed clinical signs such as depression, and the viruses were recovered from virtually all of the organs and blood samples. The remaining two did not show disease signs nor the virus was recovered from any of the tissues tested. Two of the three chickens inoculated with rgVac1ins-P2 (H5N1) showed disease signs, one of them died two days post inoculation, and the virus was recovered from almost all samples. P3 viruses efficiently replicated in each of the tested tissues in chickens as compared with P2 viruses. Throughout the study, the viruses were recovered from the brains of all of the chickens showing clinical signs.

\section{Discussion}

Here, we demonstrated that the H9N2 influenza virus acquired intravenous pathogenicity after a pair of dibasic amino acid residues was introduced into the cleavage site of the HA and serially passaged in chicks. Since rgY55sub-P10 (H9N2) killed 75\% of chickens inoculated via intravenous route, the pathogenicity was comparable to that of HPAIVs (Table 1). On the other hand, chickens intranasally inoculated with rgY55subP10 (H9N2) did not show any clinical signs of disease (Table 4). These results are consistent with those of previous study showing that some H10 influenza viruses did not show intranasal pathogenicity for chicken while their intravenous pathogenicity index was over 1.2 and classified as HPAIV according to the definition by European Union [29]. Amino acid changes during consecutive passages in the air sacs of chicks (Table 2) are considered to be responsible for the acquisition of 
Table 5 Virus recovery from the chickens intranasally inoculated with each virus

\begin{tabular}{|c|c|c|c|c|c|c|c|c|c|}
\hline \multirow[t]{2}{*}{ Inoculated viruses } & \multirow{2}{*}{$\begin{array}{c}\text { No. of } \\
\text { chickens }\end{array}$} & \multirow{2}{*}{$\begin{array}{l}\text { Days p.i. (Health } \\
\text { status) }\end{array}$} & \multicolumn{7}{|c|}{ Virus recovery $\left(\log \mathrm{EID}_{50} / \mathrm{g}\right)$} \\
\hline & & & Brain & Trachea & Lung & Liver & Kidney & Colon & Blood $^{c}$ \\
\hline $\operatorname{rgY55}(\mathrm{H} 9 \mathrm{~N} 2)$ & 3 & 3 (sacrificed) &,,$---b$ &,,$-- \leqq 1.7$ &,,--- &,,--- &,,--- &,,--- &,,--- \\
\hline rgY55sub (H9N2) & 3 & 3 (sacrificed) &,,--- & $\begin{array}{c}5.5,5.7 \\
6.5\end{array}$ & $-, 6.7,2.7$ & $-, 2.7,-$ &,,-- 2.5 &,,--- &,,--- \\
\hline $\begin{array}{l}\text { rgY55sub-P10 } \\
\text { (H9N2) }\end{array}$ & 3 & 3 (sacrificed) &,,--- &,,-- 3.3 & $-, 3.7,6.0$ &,,-- 2.5 & $-, 4.3,4.5$ & $-, 3.3,4.5$ &,,--- \\
\hline rgVac1 (H5N1) & 3 & 3 (sacrificed) &,,--- &,,--- &,,--- &,,--- &,,--- &,,--- &,,--- \\
\hline rgVac1sub (H5N1) & 3 & 3 (sacrificed) &,,-- 2.7 &,,--- &,,-- 2.5 &,,--- &,,--- &,,$-- \leqq 2.0$ &,,--- \\
\hline \multirow{2}{*}{$\begin{array}{l}\text { rgVac1sub-P2 } \\
\text { (H5N1) }\end{array}$} & 2 & 3 (sacrificed) &,-- &,-- &,-- &,-- &,-- &,-- &,-- \\
\hline & $1^{\mathrm{a}}$ & 3 (sacrificed) & 5.0 & 3.8 & 5.0 & 5.2 & 7.5 & 5.2 & 4.3 \\
\hline \multirow{2}{*}{$\begin{array}{l}\text { rgVac1sub-P3 } \\
\text { (H5N1) }\end{array}$} & $2^{\mathrm{a}}$ & 3 (dead) & $5.7,6.7$ & $5.3,6.5$ & $4.7,8.2$ & $3.5,6.0$ & $7.3,9.0$ & $5.5,6.5$ & $N A^{d}$ \\
\hline & $1^{\mathrm{a}}$ & 3 (sacrificed) & 6.3 & 6.5 & 6.7 & 6.5 & 6.5 & 6.5 & 5.8 \\
\hline rgVac1ins (H5N1) & 3 & 3 (sacrificed) &,$- \leqq 2.6$ & $-, 3.5,-$ & $-, 3.3,3.7$ &,,-- 3.5 & $-, 2.7,3.0$ & $-, 3.0,2.5$ & $\begin{array}{c}-, \leqq 1.6, \\
2.8\end{array}$ \\
\hline \multirow[t]{3}{*}{ rgVac1ins-P2 (H5N1) } & $1^{\mathrm{a}}$ & 2 (dead) & 3.5 & 3.4 & 4.7 & 3.7 & 4.8 & 4.7 & NA \\
\hline & $1^{\mathrm{a}}$ & 3 (sacrificed) & 3.8 & 3.7 & 3.0 & $\leqq 2.0$ & 4.7 & $\leqq 2.0$ & - \\
\hline & 1 & 3 (sacrificed) & - & - & - & - & - & - & - \\
\hline rgVac1ins-P3 (H5N1) & $3^{\mathrm{a}}$ & 3 (sacrificed) & $\begin{array}{l}3.4,4.7 \\
5.5\end{array}$ & $\begin{array}{l}4.7,3.5, \\
4.2\end{array}$ & $\begin{array}{l}5.5,5.2 \\
\quad 6.7\end{array}$ & $\begin{array}{c}4.3,4.5 \\
5.7\end{array}$ & $\begin{array}{l}4.5,4.7 \\
5.2\end{array}$ & $\begin{array}{l}5.3,4.5 \\
5.3\end{array}$ & $\begin{array}{c}3.0,2.5, \\
3.5\end{array}$ \\
\hline
\end{tabular}

a Each chicken showed depression.

b $1.5 \geqq(0.5 \geqq$ for blood samples).

${ }^{c} \log E I D 50 / \mathrm{ml}$.

${ }^{\mathrm{d}}$ Not applicable.

intravenous pathogenicity, and their effects on the functions of viral proteins should be clarified further. Here we focused on two substitutions at positions 29 and 234 of the H9 HA molecule. It has been reported that residue 226, based on the H3 HA numbering (234 in the present study), relates to receptor specificity and cell tropism [30]. Strain Y55 (H9N2) originally had a leucine at this position, and the change to glutamine after serial passages in the air sacs of chicks indicates that the passaged rgY55sub (H9N2) was further adapted to chicken. One of the asparagine-linked glycosylation sites on the HA of rgY55 (H9N2) lost a carbohydrate attachment with the substitution of Asn29His. The site locates sterically in the vicinity of the HA cleavage site, suggesting that the deletion of the carbohydrate chain affected the susceptibility of the HA to the host protease [31]. This notion is also supported by the present finding that the rgY55sub viruses (H9N2) after six passages in the air sacs of chicks replicated in MDCK cells in the absence of trypsin (Table 1). Ohuchi et al. (1991) reported that the insertion of additional basic amino acids into the $\mathrm{H} 3$ HA cleavage site resulted in intracellular proteolytic cleavage. Other groups reported that $\mathrm{H} 3$ and $\mathrm{H} 6 \mathrm{HAs}$ tolerated amino acid mutations into their cleavage sites and the viruses with the mutated HAs replicated in
MDCK and/or QT6 cells in the absence of trypsin $[32,33]$. The results in the present study are in agreement with these, namely, cleavage-based activation by an ubiquitous protease is not restricted to the $\mathrm{H} 5$ and H7 HAs.

rgVac1sub (H5N1) and rgVac1ins (H5N1) acquired marked intravenous and intranasal pathogenicity after a few passages in chicks (Table 1). It was reported that an avirulent $\mathrm{H} 5$ virus isolated from a swan became highly pathogenic in chickens after 24 consecutive passages in the air sacs, followed by five passages in the brains of chickens [3]. The differences in time required for the viruses to become highly pathogenic between these studies depended on the amino acid motif at the HA cleavage site prior to passaging. rgVac1sub (H5N1) acquired an arginine at the HA cleavage site after only one passage in chickens (Figure $1 \mathrm{~B}$ and Table 3 ), suggesting that an additional insertion of basic amino acid residues efficiently occurred in the serial basic amino acid residues at the cleavage site. One third of the chickens inoculated intranasally with rgVac1sub-P2 (H5N1) or rgVac1ins-P2 (H5N1) survived 14 days (Table 4). In addition, one of the birds was not susceptible to infection with rgVac1subP2 (H5N1), indicating that viral replication may 
depend on the presense of P3-like viruses in the inoculum.

The intranasal pathogenicity of the mutants of H9N2 virus was different from those of $\mathrm{H} 5 \mathrm{~N} 1$ mutants while these viruses replicated in MDCK cells in the absence of trypsin and killed chickens when inoculated via intravenous route (Tables 1 and 4). The viruses were recovered from the brain and the blood of some chickens infected with rgVac1 mutants (H5N1), and morbidity was closely associated with viral titers in the brain (Table 5). No viruses were recovered from the brain and the blood of chickens infected with rgY55 mutants (H9N2), indicating the reason why rgY55sub-P10 (H9N2) did not show intranasal pathogenicity. All the viruses passaged in the air sacs of chicks killed chicken embryos by 48 hours post allantoic inoculation (data not shown). rgVac1subP3 (H5N1) and rgVaclins-P3 (H5N1) were more pathogenic to chicken embryos than rgY55sub-P10 (H9N2); the allantoic fluids obtained from the embryonated eggs inoculated with the $\mathrm{H} 5 \mathrm{~N} 1$ viruses passaged in the air sacs were turbid. It was reported that infection of a highly pathogenic virus was strictly confined to endothelial cells in chicken embryos or chickens [34,35]. Therefore, it is suggested that the difference of endotheliotropism between the $\mathrm{H} 9 \mathrm{~N} 2$ and $\mathrm{H} 5 \mathrm{~N} 1$ viruses passaged in the air sacs affected their intranasal pathogenicity. rgY55sub-P10 (H9N2) was not recovered from the brain and the blood of chickens although it caused systemic infection (Table 5), indicating that high levels of viremia followed by replication in the vascular endothelial cells was prerequisite for the virus to cross the blood-brain barrier and consequently replicated in the brain. This hypothesis is supported by the result that rgY55sub-P10 (H9N2) showed intravenous pathogenicity in chickens; direct injection of the virus to the blood vessels readily caused viremia, leading to invasion of the virus to the brain. (Table 1).

H9N2 viruses which have the PARSKR or PARSRR motifs at their HA cleavage site have been isolated from turkeys, ostriches, and chickens in Israel and quails in China [14] although PARSSR motif has been found in most H9N2 isolates, indicating that such substitutions with basic amino acid residues occur in nature. If serine at the c-terminus of the HA1 of the H9 virus was substituted with lysine, the amino acid motif would be consistent with that of rgY55sub (H9N2) which acquired intravenous pathogenicity on consecutive passages in the air sacs of chicks. LPAI caused by H9N2 strains in poultry is now causing serious economic losses [11-19], and its eradication is still difficult because of its low pathogenicity, frequently causing inapparent infections. The present study demonstrated that H9N2 viruses circulating in chicken flocks can acquire intravenous pathogenicity. It is predicted that co-infections of rgY55sub-P10 (H9N2) with bacteria exacerbate not only intravenous pathogenicity but intranasal pathogenicity in chickens as shown in a previous study [21]. Therefore, continuous monitoring in poultry is important to prevent the emergence of pathogenic $\mathrm{H} 9$ viruses.

\section{Materials and methods}

\section{Viruses}

A/chicken/Yokohama/aq-55/2001 (Y55) (H9N2) isolated from chicken meat imported from China upon quarantine was kindly provided by Dr. M. Eto, Animal Quarantine Service (Yokohama, Kanagawa, Japan) [36]. A/duck/ Hokkaido/Vac-1/2004 (Vac1) (H5N1) was generated by the standard genetic reassortment procedure from nonpathogenic viruses, A/duck/Mongolia/54/2001 (H5N2) and A/duck/Mongolia/47/2001 (H7N1) [37-39]. Viruses were propagated in ten-day-old embryonated chicken eggs for 48 hours at $35^{\circ} \mathrm{C}$.

The complete nucleotide sequences of Y55 (H9N2) and Vac1 (H5N1) have been registered in GenBank/ EMBL/DDBJ (Accession numbers: AB256671-AB256678 [36] and AB259709-AB259716 [37], respectively).

\section{Reverse genetics}

Viral RNA was extracted from the allantoic fluid of embryonated chicken eggs infected with the Y55 and Vac1 strains using a commercial kit (TRIzol LS Reagent, Sigma-Aldrich, St. Louis, MO, U.S.A) and reversetranscribed with the Uni12 primer [40] and M-MLV Reverse Transcriptase (Invitrogen, Carlsbad, CA, U.S.A). PCR-based amplification of the full genomes of the eight gene segments was performed with universal primer sets [41]. The PCR products were cloned into the vector pCR2.1-TOPO (Invitrogen) or pGEM-T Easy Vector (Promega, Mannheim, Germany). After confirmatory sequencing, $\mathrm{T}$-vector clones were digested with $B s m B I$ and inserted into the vector pHW2000 [42]. MDCK cells and 293T cells were maintained in Minimum Essential Medium (MEM, Nissui Pharmaceutical, Tokyo, Japan) containing 10\% calf serum and D-MEM (Invitrogen) containing 10\% FBS, respectively. Before transfection, confluent $293 \mathrm{~T}$ and MDCK cells in $75 \mathrm{~cm}^{2}$ flasks were trypsinized, and $10 \%$ of each cell line was mixed in $12 \mathrm{ml}$ of Opti-MEM I (Invitrogen); $2 \mathrm{ml}$ of the suspension was seeded into each well of six-well tissue culture plates (Nunc Inc., Naperville, IL). The cocultured 293T and MDCK cells were used for the transfection. TransIT-293 (Panvera, Madison, WI) was used to transfect cells according to the manufacturer's directions. Briefly, two microliters of TransIT-293 per microgram of DNA was mixed, incubated at room temperature for 45 minutes, and added to the cells. The transfection mixture was replaced with Opti-MEM I after six hours of incubation at $37^{\circ} \mathrm{C}$. Thirty hours later, Opti-MEM I containing one microgram per microliter 
of trypsin was added. At 48 to 72 hours post-transfection, the culture supernatant was collected and propagated in ten-day-old embryonated chicken eggs.

\section{Site-directed-mutagenesis}

To generate $\mathrm{H} 9$ and $\mathrm{H} 5$ mutant viruses with basic amino acid residue substitutions (sub) or insertions (ins) at the HA cleavage site, mutations were introduced into the HA genes of the Y55 and Vac1 strains using a QuikChange II site-directed mutagenesis kit (Stratagene, Heidelberg, Germany) according to the manufacturer's instructions. The mutant viruses, rgY55sub (H9N2), rgVac1sub (H5N1), and rgVac1ins (H5N1), were rescued by reverse genetics as described above, and the entire genomes of the eight gene segments were sequenced to confirm the existence of the introduced mutations and the absence of undesired mutations.

\section{Plaque assay}

Ten-fold dilutions of viruses were inoculated onto confluent monolayers of MDCK cells and incubated at $35^{\circ} \mathrm{C}$ for one hour. Unbound viruses were removed by washing the cells with MEM. Cells were then overlaid with MEM containing 0.7\% Bacto-agar (Difco, Sparks, MD) in the presence or absence of trypsin $(5 \mu \mathrm{g} / \mathrm{ml})$. After 48 hours of incubation at $35^{\circ} \mathrm{C}$, cells were stained with $0.005 \%$ neutral red.

\section{Consecutive passage in the air sacs of chicks}

The caudal thoracic air sacs of three 3-day-old chicks were inoculated with $200 \mu \mathrm{l}$ of each of the mutant Y55 and Vac1 viruses. The chicks were sacrificed, and their lungs and brains were collected at three days post-inoculation. Serial passages in the air sacs of three to six 3-dayold chicks were performed with $200 \mu \mathrm{l}$ of a pooled $10 \%$ tissue suspension of infected organs. Brain samples were used as the inoculum when both samples (lungs and brains) tested positive for the virus. Isolates were identified by their parental strain's name, mutation (substitution or insertion), and number of passages. For example, the designation rgY55sub-P10 (H9N2) indicates that the amino acids at the HA cleavage site of the Y55 virus were substituted with basic amino acids as shown in Figure $1 \mathrm{~A}$, then passaged ten times in the air sacs. Passaged viruses were propagated in the allantoic cavities of tenday-old embryonated chicken eggs for 48 hours at $35^{\circ} \mathrm{C}$. The allantoic fluid was harvested and stored at $-80^{\circ} \mathrm{C}$.

\section{Experimental infection of chickens with mutant virus strains}

Four-week-old Boris Brown chickens were used to test the pathogenicity of the passaged viruses. Eight chickens were intravenously inoculated with $200 \mu \mathrm{l}$ of each virus (1:10 diluted allantoic fluid), and examined for clinical signs at intervals of 24 hours over a period of ten days. Similarly, three chickens were infected intranasally with $100 \mu \mathrm{l}$ of allantoic fluid containing each virus at a EID $_{50}$ of $10^{6.5}$ and observed for 14 days. Specific antibodies against homologous viruses after 14 days of infection were detected in serum using the hemagglutination inhibition (HI) test and/or enzyme-linked immunosorbent assay (ELISA) as described previously [43]. To study viral replication, each virus was inoculated into three chickens at an $\mathrm{EID}_{50}$ of $10^{6.5}$. The birds were euthenized three days post-challenge, and tissue and blood were collected aseptically. To make a $10 \%$ suspension with MEM, the tissue samples were homogenized using a Muti-Beads Shocker (Yasui Kikai, Osaka, Japan). These suspensions were serially diluted ten-fold with PBS and inoculated into ten-day-old embryonated eggs and incubated at $35^{\circ} \mathrm{C}$ for 48 hours. Viral titers were calculated by the method of Reed and Muench [44] and expressed as EID $_{50}$ per gram and milliliter of tissue and blood, respectively.

All experiments were carried out in self-contained isolator units (Tokiwa Kagaku, Tokyo, Japan) at a BSL3 biosafety facility at the Graduate School of Veterinary Medicine, Hokkaido University, Japan. The experiments were performed according to the guidelines of the institutional animal care and use committee of the Graduate School of Veterinary Medicine.

\section{Acknowledgements}

The present study was supported by the Programs of Founding Research Centers for Emerging and Re-Emerging Infectious Diseases from the Ministry of Education, Culture, Sports, Science and Technology, Japan, and SolutionOriented Research for Science and Technology (SORST) from the Japan Science and Technology Agency (JST).

\section{Author details}

'Laboratory of Microbiology, Department of Disease Control, Graduate School of Veterinary Medicine, Hokkaido University, Sapporo, Hokkaido 0600818, Japan. ${ }^{2}$ Research Center for Zoonosis Control, Hokkaido University, Sapporo, Hokkaido 001-0020, Japan. ${ }^{3}$ SORST, Japan Science and Technology Agency (JST), 4-1-8 Honcho Kawaguchi, Saitama 331-0012, Japan.

\section{Authors' contributions}

HK is the leader of the study group. KS carried out the experiments and wrote the manuscript. SA helped in passaging study. KS, MO, YS, and HK designed the experiments and analyzed the data. All authors read and approved the final manuscript.

\section{Competing interests}

The authors declare that they have no competing interests.

Received: 24 November 2010 Accepted: 10 February 2011 Published: 10 February 2011

\section{References}

1. Fouchier RA, Munster V, Wallensten A, Bestebroer TM, Herfst S, Smith D, Rimmelzwaan GF, Olsen B, Osterhaus AD: Characterization of a novel influenza $A$ virus hemagglutinin subtype $(\mathrm{H} 16)$ obtained from blackheaded gulls. J Virol 2005, 79:2814-2822.

2. Chen H, Deng G, Li Z, Tian G, Li Y, Jiao P, Zhang L, Liu Z, Webster RG, Yu K: The evolution of H5N1 influenza viruses in ducks in southern China. Proc Natl Acad Sci USA 2004, 101:10452-10457. 
3. Ito T, Goto H, Yamamoto E, Tanaka H, Takeuchi M, Kuwayama M, Kawaoka Y, Otsuki K: Generation of a highly pathogenic avian influenza A virus from an avirulent field isolate by passaging in chickens. J Virol 2001, 75:4439-4443.

4. Makarova NV, Ozaki H, Kida H, Webster RG, Perez DR: Replication and transmission of influenza viruses in Japanese quail. Virology 2003, 310:8-15.

5. Perez DR, Lim W, Seiler JP, Yi G, Peiris M, Shortridge KF, Webster RG: Role of quail in the interspecies transmission of $\mathrm{H} 9$ influenza $A$ viruses: molecular changes on HA that correspond to adaptation from ducks to chickens. J Virol 2003, 77:3148-3156.

6. Tumpey TM, Kapczynski DR, Swayne DE: Comparative susceptibility of chickens and turkeys to avian influenza A H7N2 virus infection and protective efficacy of a commercial avian influenza H7N2 virus vaccine. Avian Dis 2004, 48:167-176

7. Senne DA, Panigrahy B, Kawaoka Y, Pearson JE, Suss J, Lipkind M, Kida H, Webster RG: Survey of the hemagglutinin (HA) cleavage site sequence of $\mathrm{H} 5$ and $\mathrm{H} 7$ avian influenza viruses: amino acid sequence at the HA cleavage site as a marker of pathogenicity potential. Avian Dis 1996, 40:425-437.

8. Ito T, Kawaoka Y: Avian influenza. In Textbook of Influenza. Edited by: Nicholson KG, Webster RG, Hay AJ. Blackwell Science Ltd, Oxford; 1998:126-136.

9. Decha P, Rungrotmongkol T, Intharathep P, Malaisree M, Aruksakunwong O, Laohpongspaisan C, Parasuk V, Sompornpisut P, Pianwanit S, Kokpol S, Hannongbua S: Source of high pathogenicity of an avian influenza virus H5N1: why H5 is better cleaved by furin. Biophys J 2008, 95:128-134

10. Guo XL, Li L, Wei DQ, Zhu YS, Chou KC: Cleavage mechanism of the H5N1 hemagglutinin by trypsin and furin. Amino Acids 2008, 35:375-382.

11. Li KS, Xu KM, Peiris JS, Poon LL, Yu KZ, Yuen KY, Shortridge KF, Webster RG, Guan $Y$ : Characterization of $\mathrm{H} 9$ subtype influenza viruses from the ducks of southern China: a candidate for the next influenza pandemic in humans? J Virol 2003, 77:6988-6994

12. Liu JH, Okazaki K, Mweene A, Shi WM, Wu QM, Su JL, Zhang GZ, Bai GR, Kida $\mathrm{H}$ : Genetic conservation of hemagglutinin gene of $\mathrm{H} 9$ influenza virus in chicken population in Mainland China. Virus Genes 2004 , 29:329-334.

13. Lee $Y J$, Shin JY, Song MS, Lee YM, Choi JG, Lee EK, Jeong OM, Sung HW, Kim JH, Kwon YK, Kwon JH, Kim CJ, Webby RJ, Webster RG, Choi YK: Continuing evolution of $\mathrm{H} 9$ influenza viruses in Korean poultry. Virology 2007, 359:313-323.

14. Xu KM, Li KS, Smith GJ, Li JW, Tai H, Zhang JX, Webster RG, Peiris JS Chen $\mathrm{H}$, Guan Y: Evolution and molecular epidemiology of H9N2 influenza A viruses from quail in southern China, 2000 to 2005. J Virol 2007, 81:2635-2645.

15. Guo YJ, Krauss S, Senne DA, Mo IP, Lo KS, Xiong XP, Norwood M, Shortridge KF, Webster RG, Guan Y: Characterization of the pathogenicity of members of the newly established H9N2 influenza virus lineages in Asia. Virology 2000, 267:279-288.

16. Lee CW, Song CS, Lee YJ, Mo IP, Garcia M, Suarez DL, Kim SJ: Sequence analysis of the hemagglutinin gene of H9N2 Korean avian influenza viruses and assessment of the pathogenic potential of isolate MS96. Avian Dis 2000, 44:527-535

17. Guan Y, Shortridge KF, Krauss S, Webster RG: Molecular characterization of H9N2 influenza viruses: were they the donors of the "internal" genes of H5N1 viruses in Hong Kong? Proc Natl Acad Sci USA 1999, 96:9363-9367.

18. Guan Y, Shortridge KF, Krauss S, Chin PS, Dyrting KC, Ellis TM, Webster RG, Peiris M: H9N2 influenza viruses possessing $\mathrm{H} 5 \mathrm{~N} 1$-like internal genomes continue to circulate in poultry in southeastern China. J Virol 2000, 74:9372-9380

19. Haghighat-Jahromi M, Asasi $\mathrm{K}$, Nili H, Dadras H: Role of infectious bronchitis live vaccine on pathogenicity of H9N2 avian influenza virus. Int J Poult Sci 2007, 6:838-841.

20. Mo IP, Song CS, Kim KS, Rhee JC: An occurrence of non-highly pathogenic avian influenza in Korea. Proc 4th International Symposium on Avian Influenza, Athens, GA 1998, 379-383.

21. Kishida N, Sakoda Y, Eto M, Sunaga Y, Kida H: Co-infection of Staphylococcus aureus or Haemophilus paragallinarum exacerbates H9N2 influenza A virus infection in chickens. Arch Virol 2004, 149:2095-2104.
22. Bano S, Naeem K, Malik SA: Evaluation of pathogenic potential of avian influenza virus serotype H9N2 in chickens. Avian Dis 2003, 47:817-822.

23. Haghighat-Jahromi M, Asasi K, Nili H, Dadras H, Shooshtari AH: Coinfection of avian influenza virus (H9N2 subtype) with infectious bronchitis live vaccine. Arch Virol 2008, 153:651-655.

24. Peiris M, Yuen KY, Leung CW, Chan KH, Ip PL, Lai RW, Orr WK, Shortridge KF: Human infection with influenza H9N2. Lancet 1999, 354:916-917

25. Peiris JS, Guan Y, Markwell D, Ghose P, Webster RG, Shortridge KF: Cocirculation of avian H9N2 and contemporary "human" H3N2 influenza A viruses in pigs in southeastern China: potential for genetic reassortment? J Virol 2001, 75:9679-9686.

26. Maines TR, Szretter KJ, Perrone L, Belser JA, Bright RA, Zeng H, Tumpey TM, Katz JM: Pathogenesis of emerging avian influenza viruses in mammals and the host innate immune response. Immunol Rev 2008, 225:68-84.

27. Saito T, Lim W, Suzuki T, Suzuki Y, Kida H, Nishimura SI, Tashiro M: Characterization of a human H9N2 influenza virus isolated in Hong Kong. Vaccine 2001, 20:125-133.

28. OIE: Avian influenza, Manual of Diagnostic Tests and Vaccines For Terrestrial Animals (mammals, birds and bees). Sixth 2008, 1:465-481.

29. Wood GW, Banks J, Strong I, Parsons G, Alexander DJ: An avian influenza virus of $\mathrm{H} 10$ subtype that is highly pathogenic for chickens, but lacks multiple basic amino acids at the haemagglutinin cleavage site. Avian Pathol 1996, 25:799-806.

30. Wan H, Perez DR: Amino acid 226 in the hemagglutinin of H9N2 influenza viruses determines cell tropism and replication in human airway epithelial cells. J Virol 2007, 81:5181-5191.

31. Webster RG, Kawaoka Y, Bean WJ Jr: Molecular changes in A/Chicken/ Pennsylvania/83 (H5N2) influenza virus associated with acquisition of virulence. Virology 1986, 149:165-173.

32. Munster VJ, Schrauwen EJ, de Wit E, van den Brand JM, Bestebroer TM, Herfst S, Rimmelzwaan GF, Osterhaus AD, Fouchier RA: Insertion of a multibasic cleavage motif into the hemagglutinin of a low-pathogenic avian influenza $\mathrm{H} 6 \mathrm{~N} 1$ virus induces a highly pathogenic phenotype. J Virol 2010, 84:7953-7960.

33. Stech O, Veits J, Weber S, Deckers D, Schroer D, Vahlenkamp TW Breithaupt A, Teifke J, Mettenleiter TC, Stech J: Acquisition of a polybasic hemagglutinin cleavage site by a low-pathogenic avian influenza virus is not sufficient for immediate transformation into a highly pathogenic strain. J Virol 2009, 83:5864-5868.

34. van Riel D, van den Brand J, Munster VJ, Besteboer TM, Fouchier RA, Osterhaus A, Kuiken T: Pathology and Virus Distribution in Chickens Naturally Infected with Highly Pathogenic Avian Influenza a Virus (H7N7) During the 2003 Outbreak in the Netherlands. Vet Pathol 2009, 46:971-976.

35. Feldmann A, Schafer MK, Garten W, Klenk HD: Targeted infection of endothelial cells by avian influenza virus A/FPV/Rostock/34 (H7N1) in chicken embryos. J Virol 2000, 74:8018-8027.

36. Mase M, Eto M, Imai K, Tsukamoto K, Yamaguchi S: Characterization of H9N2 influenza A viruses isolated from chicken products imported into Japan from China. Epidemiol Infect 2007, 135:386-391.

37. Soda K, Sakoda Y, Isoda N, Kajihara M, Haraguchi Y, Shibuya H, Yoshida H, Sasaki T, Sakamoto R, Saijo K, Hagiwara J, Kida H: Development of vaccine strains of $\mathrm{H} 5$ and H7 influenza viruses. Jpn J Vet Res 2008, 55:93-98.

38. Soda K, Ozaki H, Sakoda Y, Isoda N, Haraguchi Y, Sakabe S, Kuboki N, Kishida N, Takada A, Kida H: Antigenic and genetic analysis of $\mathrm{H} 5$ influenza viruses isolated from water birds for the purpose of vaccine use. Arch Virol 2008, 153:2041-2048.

39. Isoda N, Sakoda Y, Kishida N, Soda K, Sakabe S, Sakamoto R, Imamura T, Sakaguchi M, Sasaki T, Kokumai N, Ohgitani T, Saijo K, Sawata A, Hagiwara J, Lin Z, Kida H: Potency of an inactivated avian influenza vaccine prepared from a non-pathogenic $\mathrm{H} 5 \mathrm{~N} 1$ reassortant virus generated between isolates from migratory ducks in Asia. Arch Virol 2008, 153:1685-1692.

40. Desselberger U, Racaniello VR, Zazra JJ, Palese P: The $3^{\prime}$ and $5^{\prime}$-terminal sequences of influenza $A, B$ and $C$ virus RNA segments are highly conserved and show partial inverted complementarity. Gene 1980, 8:315-328.

41. Hoffmann E, Stech J, Guan Y, Webster RG, Perez DR: Universal primer set for the full-length amplification of all influenza A viruses. Arch Virol 2001, $146: 2275-2289$ 
42. Hoffmann E, Neumann G, Kawaoka Y, Hobom G, Webster RG: A DNA transfection system for generation of influenza A virus from eight plasmids. Proc Natl Acad Sci USA 2000, 97:6108-6113.

43. Kida $H$, Brown $L E$, Webster RG: Biological activity of monoclonal antibodies to operationally defined antigenic regions on the hemagglutinin molecule of A/Seal/Massachusetts/1/80 (H7N7) influenza virus. Virology 1982, 122:38-47.

44. Reed M, Muench $\mathrm{H}$ : A simple method for estimating fifty percent endpoints. Am J Hyg 1938, 37:493-497.

doi:10.1186/1743-422X-8-64

Cite this article as: Soda et al:: H9N2 influenza virus acquires

intravenous pathogenicity on the introduction of a pair of di-basic amino acid residues at the cleavage site of the hemagglutinin and consecutive passages in chickens. Virology Journal 2011 8:64.

Submit your next manuscript to BioMed Central and take full advantage of:

- Convenient online submission

- Thorough peer review

- No space constraints or color figure charges

- Immediate publication on acceptance

- Inclusion in PubMed, CAS, Scopus and Google Scholar

- Research which is freely available for redistribution

Submit your manuscript at www.biomedcentral.com/submit 